\title{
MiniBooNE: Status of the Booster Neutrino Experiment
}

\author{
Andrew O. Bazarko for the MiniBooNE Collaboration * \\ Department of Physics, Princeton University, Princeton, New Jersey 08544-0708, USA
}

MiniBooNE is preparing to search for $\nu_{\mu} \rightarrow \nu_{e}$ oscillations at Fermilab. The experiment is designed to make a conclusive statement about LSND's neutrino oscillation evidence. We give a status report on the preparation of the experiment and outline the experimental prospects.

\section{INTRODUCTION}

There are now three reported indications for neutrino oscillations. The most compelling evidence comes from experiments studying neutrinos produced by cosmic rays in the earth's atmosphere, which find that muon neutrinos $\left(\nu_{\mu}\right)$ entering the detector from below (which had to travel through the earth) are depleted when compared with those incident from above (which traveled a shorter distance) [1]. There is also evidence from solar neutrino experiments, which observe fewer electron neutrinos $\left(\nu_{e}\right)$ than would be consistent with the sun's energy output [2]. Finally, there is evidence from a lone accelerator experiment, LSND at Los Alamos, which observed an excess of $\bar{\nu}_{e}$ events from a predominantly $\bar{\nu}_{\mu}$ beam [3.

The full picture of neutrino oscillations remains incomplete. The three reported indications differ according to amplitude, frequency, and what neutrino flavors participate, and cannot be explained by oscillations involving only the three known neutrino flavors. Perhaps further study will reveal a fourth neutrino.

MiniBooNE is in a unique position to help address this possibility, because MiniBooNE is alone among upcoming experiments with sensitivity to the LSND mode of oscillations. MiniBooNE is designed to confirm or refute LSND unequivocally, and thereby either keep alive or kill evidence for a fourth neutrino. The MiniBooNE Collabora-

\footnotetext{
${ }^{*}$ To appear in Proceedings of the XIX International Conference on Neutrino Physics and Astrophysics (Neutrino 2000), Sudbury, Canada, 16-21 June 2000.
}

tion is now building a new beam and detector at Fermilab, and we plan to start collecting data in December 2001.

\section{EXPERIMENTAL OVERVIEW}

At MiniBooNE we will search for the oscillation of muon neutrinos into electron neutrinos $\left(\nu_{\mu} \rightarrow \nu_{e}\right)$. We will produce a $\nu_{\mu}$ beam in the energy range $0.5-1.0 \mathrm{GeV}$ with a small intrinsic $\nu_{e}$ component (less than $0.3 \%$ ) and search for an excess of electron neutrino events in a detector located approximately $500 \mathrm{~m}$ from the neutrino source. The baseline to neutrino energy ratio will thereby be similar to that of LSND, $L / E \sim 1$, giving MiniBooNE sensitivity to the same mode of oscillations. However, neutrino energies will be more than an order of magnitude higher than at LSND, so that the search at MiniBooNE will be in a different environment and will employ different experimental signatures.

The MiniBooNE neutrino beam will be initiated by a primary beam of $8 \mathrm{GeV}$ protons from the Fermilab Booster. The Booster is a reliable, high intensity machine, expected to run at least $2 \times 10^{7}$ s per year, while delivering $5 \times 10^{12}$ protons per $1.6 \mu$ s pulse at a rate of $5 \mathrm{~Hz}$ to MiniBooNE. One year for the experiment is expected to correspond to $5 \times 10^{20}$ protons on target. The Booster has the capacity to provide protons for several Fermilab efforts, and when MiniBooNE starts, the Booster will supply beam to both the Tevatron collider and to MiniBooNE. Later, we anticipate that the Tevatron, NuMI, and MiniBooNE programs can be accommodated simulta- 


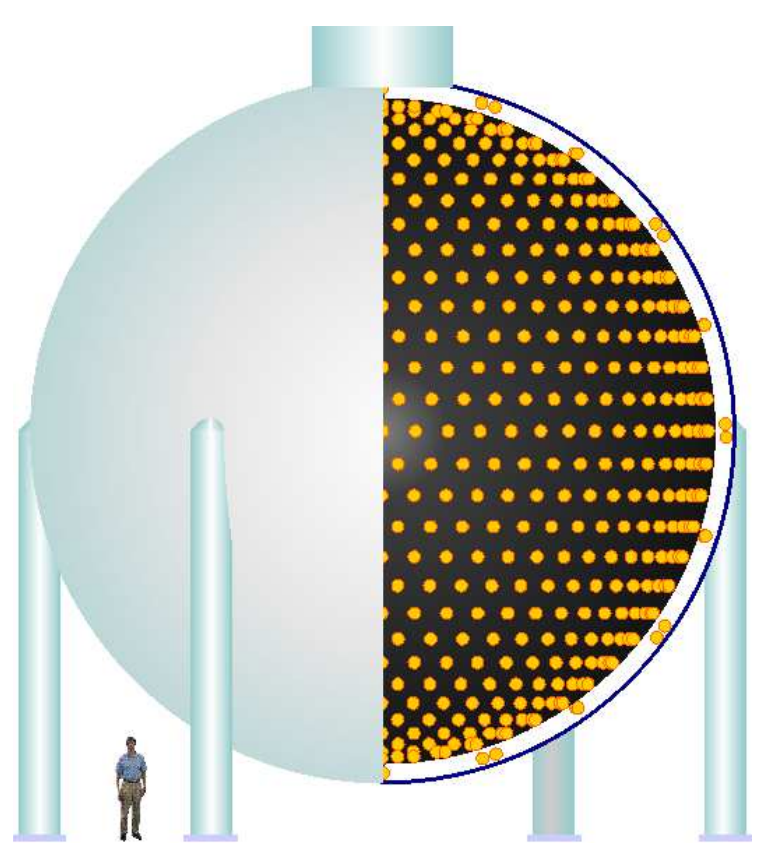

Figure 1. A schematic of the MiniBooNE detector. The cutaway shows the layout of 8-inch phototubes in the black main region, and in the white veto region.

neously.

A secondary beam will be produced when the 8 $\mathrm{GeV}$ protons strike a beryllium target positioned inside a magnetic horn. Positively charged particles (mostly pions) from the target will be focused forward by the single horn into a $50 \mathrm{~m}$ decay channel. Decays of these secondaries will constitute the neutrino beam. Decay lengths of 50 and $25 \mathrm{~m}$ will be possible through the use of two steel and concrete beam absorbers. One absorber will be permanently positioned at the end of the decay channel. The intermediate absorber is designed either to be lowered into the decay channel or to be raised out of the way. This ability to vary the decay length will provide a check of experimental systematics associated with $\nu_{e}$ contamination.

The MiniBooNE neutrino detector will consist of 800 tons of pure mineral oil contained in a 40foot $(12.2 \mathrm{~m})$ diameter spherical tank. A structure in the tank will support phototubes, which will detect neutrino interactions in the oil by the Cherenkov and scintillation light that they produce. (The undoped mineral oil will scintillate modestly from the presence of intrinsic impurities.) The phototube support structure will also optically isolate the most outer $35 \mathrm{~cm}$ of oil from the rest, turning the outer oil into a veto region that should stay quiet while a neutrino produces light only in the inner, main region. The main region will be viewed by 12808 -inch phototubes, providing $10 \%$ photocathode coverage of the 445 ton fiducial volume. The veto region will contain 240 8-inch phototubes mounted in pairs on the tank wall. In order to limit reflections, main volume surfaces will be black in color. Surfaces in the veto region, including the tank wall, will be white, in order to maximize light collection. A schematic of the detector is shown in Fig. 1.

The center of the detector will be positioned about $490 \mathrm{~m}$ from the end of the decay channel, and about $6 \mathrm{~m}$ below ground, corresponding to the level of the neutrino beam. The tank will be housed in a cylindrical concrete vault. A room above the vault will contain the experiment's electronics. Approximately $3 \mathrm{~m}$ of soil will be placed on top of the enclosure with access through a corridor, as shown in Fig. 2.

\section{STATUS}

A few of MiniBooNE's features have changed from those described in the proposal [4]. Most notable is the switch from two-horn secondary beam focusing to a single horn design.

Much work is ongoing to allow MiniBooNE to start running in December 2001. The status on several fronts as of August 2000 is reported here.

\subsection{Civil construction}

Construction of the detector enclosure is nearing completion. Pieces of the steel tank arrived at Fermilab in early April and were assembled inside the detector vault. In June, the completed tank was successfully hydrotested, that is, it was filled with water, subjected to a slight overpressure, and found not to leak. Since then, the electronics room has been completed and the earth berm up to the roof of the enclosure has been 


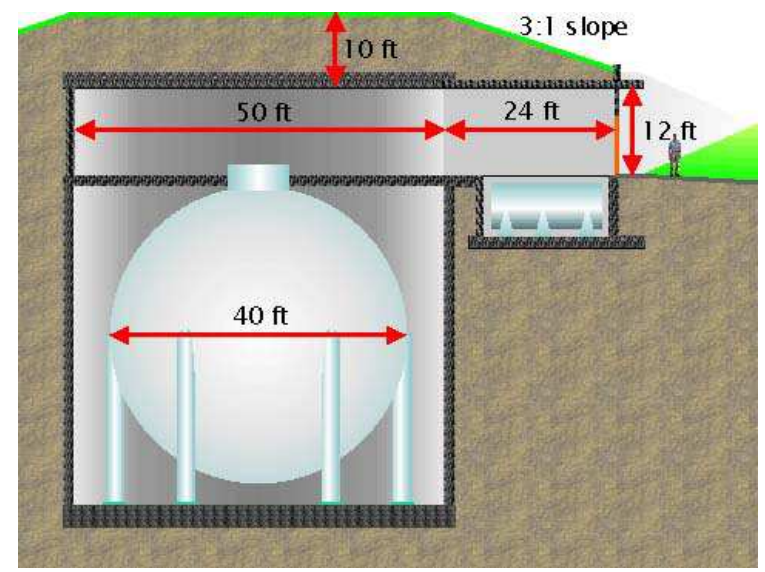

Figure 2. Cutaway of the underground MiniBooNE detector enclosure. An oil overflow tank is shown underneath the corridor.

constructed. The tank is being painted and the enclosure is being outfitted with utilities. We experimenters expect to take occupancy of the space in October.

Construction of the $8 \mathrm{GeV}$ beam, the target hall, and the decay channel began in July. Concrete at the foundation of the target hall is in place. The target hall walls are to go up next, and an opening in one wall will lead to the decay channel. The decay channel will be enclosed in a waterproof liner, to prevent migration of radioactivity from the soil near the channel. The liner material remains pliable down to a few degrees Celsius, so it cannot be installed during the cold of winter. Therefore, an important item on the beam construction project schedule is liner installation by late October, which appears feasible.

\subsection{Detector components}

MiniBooNE will reuse the phototubes from LSND together with 330 new tubes acquired from Hamamatsu. LSND's 1220 phototubes are now at Fermilab, and shipments of the new tubes have been arriving over the summer. Both new and old tubes are being tested to characterize charge and time resolutions and to determine operat- ing voltages. Results for the LSND tubes correspond nicely with old measurements, indicating that these old tubes remain stable after their journey from New Mexico to Illinois. Tests also verify the better performance of the new tubes, which have a greater number of dynode stages and narrower time resolution.

A prototype of the phototube support structure has been assembled and final components are being fabricated. The bulk of this aluminum structure is being painted, black on one side (to face the main volume) and white on the other (to face the veto). Aircraft paints have been chosen. The white offers high albedo over most of the visible spectrum, whereas the black is quite nonreflective, even at large angles of incidence. These paints offer good adhesion to aluminum and our tests indicate that they will not contaminate the mineral oil. Some smaller aluminum pieces used to mount phototubes in the main region will be sandblasted and black anodized, a process that also meets our low-albedo and low-contamination requirements.

Preparation of the cables that will connect the phototubes to pre-amplifier cards outside the tank has started. Each phototube will be attached to one cable that will both supply the high voltage and carry out the signal. Cables will be routed along the wall of the tank, and they will penetrate individually into the main volume as needed. Because these cables will run through the veto region, they will be jacketed in white, and teflon jacket material has been chosen because it is non-contaminating (our tests indicate that standard PVC cable jackets contaminate mineral oil). Each phototube will have a short "pigtail" of black cable to which the white cable will be spliced. Thus black cable will be in the main volume and white cable will be in the veto region.

Work is ongoing on other parts of the data acquisition system. Along with the phototubes, the charge and time readout electronics from LSND, the so-called QT cards and crates, will be reused. New high voltage power supplies are being acquired, and new event building hardware and software are being developed. Progress is also being made on calibration systems. A laser calibration system will provide short pulses of light from 
a tunable dye laser to four light-diffusing flasks at various locations in the detector. This system is similar to one used successfully in LSND to determine phototube time offsets and time slewing corrections. In addition, a cosmic ray calibration system is being built. This system will consist of a muon tracker (scintillator hodoscope) above the detector with scintillator cubes $(5 \mathrm{~cm}$ on a side) located under the tracker inside the detector. The system will provide the entering position and direction of cosmic ray muons that stop in the detector, including the fraction that stop in a cube. These events will be used to calibrate the position, energy, and direction determination of the reconstruction algorithm.

A scaffold will be constructed inside the tank for installation of the phototubes and the other detector internals. Phototube installation is expected to take four to five months and should begin in January 2001. Filling the tank with mineral oil is planned for fall 2001 .

\subsection{Beam components}

An array of components - magnets, power supplies, vacuum systems, water cooling systems, beam monitors, collimators, etc. — is being prepared to transport the $8 \mathrm{GeV}$ protons from the Booster to the target hall and form the neutrino beam. The most challenging element to construct is the magnetic horn that will focus the secondary beam.

The MiniBooNE horn is designed to operate with $170 \mathrm{kA}$ pulses at a rate of $5 \mathrm{~Hz}$, with the goal that it withstand 200 Mpulses (two years of operation). This is a higher repetition rate and more pulses than any previous horn. The horn is designed to tolerate the stresses that each pulse will bring as the current heats the conductors and generates magnetic forces. The heat will be managed with water cooling, and the horn body is designed to contain the radioactive water that will circulate inside. Numerous stress calculations have been performed, with concern over such issues as metal fatigue, structural resonances, and water erosion. In the end, however, tests on a prototype will settle the design.

Several horn and target pre-prototype tests have been completed. For example, cooling tests for the $65 \mathrm{~cm}$ air-cooled beryllium target were completed, and target construction is on track. Various elements of the horn and its power supply have been tested, such as the water cooling system, the welding procedures, and the possibility of excessive corona currents in the design of high potential difference areas. Parts for a prototype horn are being procured, and a proto-horn will be assembled over the winter, with tests scheduled for spring 2001. The plan is to test the proto-horn for at least 10 Mpulses, which will mark an important milestone in the beamline construction. Ultimately, we plan to have an installed horn and a spare, in addition to the proto-horn.

The target and horn will be contained under a pile of steel and concrete shielding in the target hall. "Hot horn handling" procedures are being planned to limit exposures to radioactivity should horn replacement be necessary. These involve putting the radioactive target and horn assembly into a "coffin" for safe removal from the target hall.

\section{OUTLINE OF FUTURE ANALYSIS}

We will reconstruct quasielastic $\nu_{e}$ interactions by identifying electrons via their characteristic Cherenkov and scintillation light signatures. Besides the $\nu_{e} \rightarrow e^{-}$signal, several backgrounds will contribute. The analysis will come down to accounting for the backgrounds and determining whether or not there is an excess. The background sources will be due to $\nu_{e}$ contamination in the beam and to the misidentification as electrons of muons and $\pi^{0}$ 's produced in the detector. Because the neutrinos are at higher energies than at LSND, neutrons will not play a role in the signal and will not contribute background.

The detector will record the time of the initial hit and total charge for each phototube. From this information, the track position and direction will be determined. Muon tracks will be distinguished from electron tracks by their Cherenkov rings and scintillation light. Electrons will tend to produce "fuzzy" rings due to multiple scattering and bremsstrahlung, while muon rings will tend to have sharp outer boundaries. Electrons also tend to have a high fraction of prompt 
(Cherenkov) light compared to late (scintillation) light, whereas muons produce relatively more late light.

MiniBooNE's secondary beam will be dominated by pions $\left(\pi^{+}\right)$with energies around $2 \mathrm{GeV}$. Pions decay to a $\nu_{\mu}$ and a muon $99.988 \%$ of the time. Most of the muons $\left(\mu^{+}\right)$, because of their relatively long lifetime, will travel to the end of the decay channel and stop in the absorber or the dirt. Any muon that decays, however, will yield a $\nu_{e}$. So, pions are an almost pure source of $\nu_{\mu}$ 's, but the muons that are produced along with them contribute some $\nu_{e}$ contamination. This situation is not as bad as it might seem, because both the muon and the $\nu_{\mu}$ emerge from the same two-body pion decay, which allows the measurement of one of the decay particles to constrain the other. In one year of running, the MiniBooNE detector will measure about 0.5 million $\nu_{\mu}$ events. These $\nu_{\mu}$ events will act as excellent monitors of the pions that produced them, because the two-body kinematics, relatively low beam energy, and the small angle subtended by the detector conspire to produce a very tight correlation between $\pi^{+}$and $\nu_{\mu}$ energies. Thus, the pion secondary beam will be well constrained, and then so too will be the $\pi^{+} \rightarrow \mu^{+} \rightarrow \nu_{e}$ chain. In this way, we expect to reduce the uncertainty in the muon component of the $\nu_{e}$ background to less than $5 \%$.

While pions will comprise most of the secondary beam, some kaons will also be produced at the target. Charged kaons decay via $K^{+} \rightarrow$ $\pi^{0} e^{+} \nu_{e} 4.8 \%$ of the time, and neutral kaons decay via $K_{L} \rightarrow \pi^{ \pm} e^{\mp} \nu_{e} 38.8 \%$ of the time. With many fewer kaons than pions produced, the overall kaon contribution to $\nu_{e}$ contamination is expected to be about one third of that due to the muons. Because the $K^{+}$'s are focused by the horn and the $K_{L}$ 's are not, the $K^{+} \nu_{e}$ component will be about twice that of the $K_{L}$. We plan to use Monte Carlo simulation constrained by production data to limit the systematic uncertainty in the kaon component of the $\nu_{e}$ background to about $10 \%$. We continue to explore ways to directly measure the kaon content of our secondary beam.

The number of muons that will be misidentified as electrons by the reconstruction algorithm can be estimated using the data. Ninety two per- cent of the muons contained in the detector will decay, and they will be relatively easily identified by the presence of a second track (the decay electron). However, the $8 \%$ of muons that get captured have a greater chance to be misidentified. The misidentification of muon captures will be estimated by studying the large sample of muons that decay and determining the particle identification algorithm performance while ignoring the decay track. Using this technique, which does not rely on Monte Carlo simulation, the muon misidentification uncertainty is expected to be below $5 \%$.

Most neutral pions will be identified by their two electromagnetic decay tracks. A small fraction (1\%) of asymmetric $\pi^{0}$ decays will not yield two resolvable tracks and will therefore be more likely to be misidentified. The misidentification contribution of these decays will be studied with Monte Carlo simulation, which will be constrained by the large sample of measured $\pi^{0}$ 's in the experiment. The pion misidentification uncertainty is expected to be $5 \%$.

\section{PROSPECTS}

If oscillations occur as indicated by LSND, MiniBooNE will observe an excess of several hundred electron events in one year of running. The significance of this excess would be on the order of 8 to $10 \sigma$ above background expectations.

In addition to a count of excess events, we will be able to measure their energy distribution, and thereby enhance the experimental sensitivity. The expected energy spectra for excess events in two oscillation scenarios are shown in Fig. 3. Backgrounds are expected to have different energy distributions from the oscillation events, so an underestimate of the background will not necessarily be interpreted as a fictitious oscillation signal.

Fig. 4 shows MiniBooNE's expected exclusion contours.

\section{REFERENCES}

1. H. Sobel, these proceedings, and SuperKamiokande Collab., Y. Fukuda et al., 

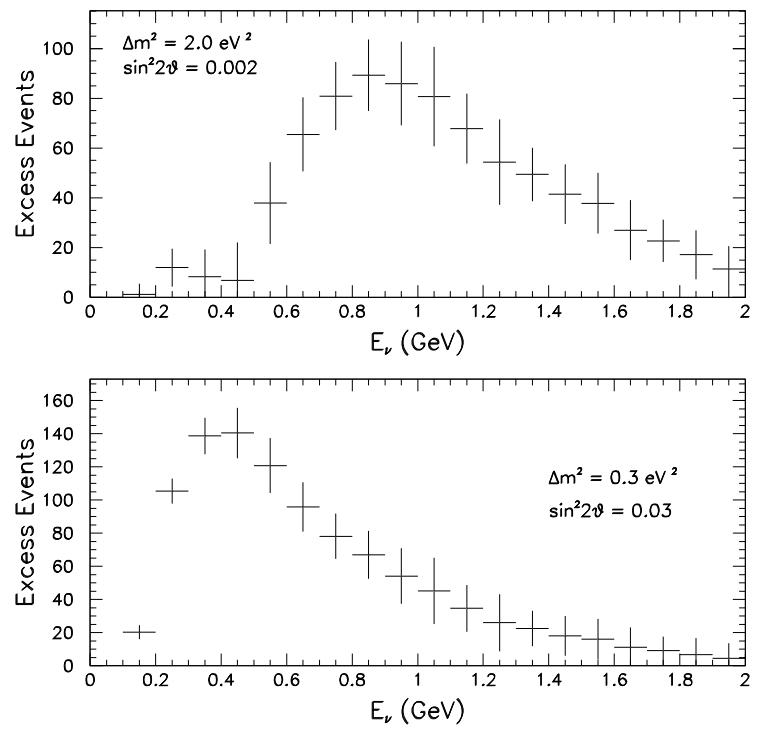

Figure 3. Energy spectra of the $\nu_{e}$ event excess expected for two oscillation scenarios. The excess for $\Delta m^{2}=2 \mathrm{eV}^{2}, \sin ^{2} 2 \theta=0.002$ is shown in the upper plot and that for $\Delta m^{2}=0.3 \mathrm{eV}^{2}, \sin ^{2} 2 \theta=$ 0.03 is in the lower plot. The error bars include both statistical and systematic uncertainties, the latter of which are correlated from bin to bin.

Phys. Rev. Lett. 81 (1998) 1562-1567.

B. Barish, these proceedings, and

MACRO Collab., M. Ambrosio et al., Phys. Lett. B 478 (2000) 5-13.

W.A. Mann, these proceedings, Soudan-2 Collab.

2. J. N. Bahcall, R. Davis, et al., eds. Solar Neutrinos: The first thirty years. (Reading, USA: Addison-Wesley) 1995.

Y. Suzuki, these proceedings, and

SuperKamiokande Collab., Y. Fukuda et al., Phys. Rev. Lett. 82 (1999) 2430-2434.

V. Gavrin, these proceedings, and SAGE Collab., J.N. Abdurashitov et al., Phys. Rev. Lett. 83 (1999) 4686-4689.

E. Bellotti, these proceedings, and

GALLEX Collab., Phys. Lett. B 447 (1999) 127
3. G. Mills, these proceedings, and

LSND Collab., C. Athanassopoulos et al., Phys. Rev. Lett. 81 (1998) 1774-1777.

4. See www-boone.fnal.gov. The MiniBooNE proposal is available at www.neutrino.lanl.gov/BooNE/ boone_proposal.ps

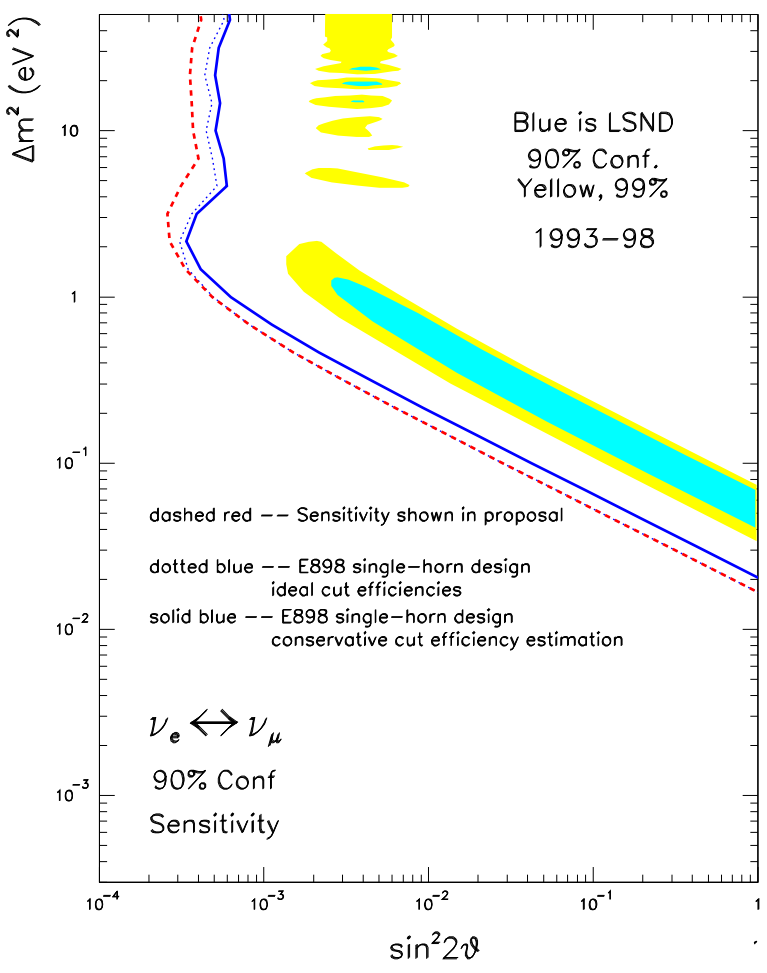

Figure 4. MiniBooNE expected $90 \%$ confidence level sensitivity limits. The solid curve is the expectation for the final single horn design and an analysis with conservative efficiency assumptions. The dotted curve is for the single horn and ideal efficiency. The dashed curve is the sensitivity using a two-horn design as described in the experiment's proposal. 